\title{
The impact of nonlinear perturbation to the dynamics of HIV model
}

\author{
Zhenfeng Shi ${ }^{1}$, Daqing Jiang ${ }^{2}$, Ningzhong Shi ${ }^{3}$, Tasawar Hayat ${ }^{4}$, and Ahmed Alsaedi ${ }^{5}$ \\ ${ }^{1}$ Northeast Normal University \\ ${ }^{2}$ College of Science, China University of Petroleum(East China) \\ ${ }^{3}$ School of Mathematics and Statistics, Northeast Normal University \\ ${ }^{4}$ Quaid-i-Azam University \\ ${ }^{5}$ King Abdulaziz University
}

March 15, 2021

\begin{abstract}
In this paper, we developed and studied a stochastic HIV model with nonlinear perturbation. Through a rigorous analysis, we firstly showed that the solution of the stochastic model is positive and global. Then, by employing suitable stochastic Lyapunov functions, we prove that the stochastic model admit a unique ergodic stationary distribution. In addition, sufficient conditions for the extinction of HIV infection are derived. Finally, numerical simulations are employed to confirm our theoretical results.
\end{abstract}

\section{Hosted file}

HIVmodel.pdf available at https://authorea.com/users/401703/articles/513703-the-impact-ofnonlinear-perturbation-to-the-dynamics-of-hiv-model 
figures/Fig1/Fig1-eps-converted-to.pdf 
figures/Fig2/Fig2-eps-converted-to.pdf 